\title{
HIPODONCIA: REPORTE DE UN CASO FAMILIAR
}

\author{
Gloria Cristina Aránzazu Moya \\ Odontóloga U. Santo Tomás, Especialista en Patología Oral y Medios Diagnósticos U. El Bosque, Docente U. Santo Tomás
}

Dirección de correo electrónico:aranzazugloria@yahoo.es

\begin{abstract}
RESUMEN
La hipodoncia se define como la falta de uno a seis dientes, sin tener en cuenta a los terceros molares; es un evento que ocurre, en la dentición permanente, entre el $1.6 \%$ al $9.6 \%$ tanto en hombres como en mujeres. Se reporta un caso de hipodoncia familiar con un patrón de herencia autosómico recesivo, con ausencia de incisivo lateral izquierdo y segundos premolares en los cuatro cuadrantes, además de los terceros molares y microdoncia de incisivo lateral derecho. [Aránzazu GC. Hipodoncia: Reporte de un caso familiar. Revista Ustasalud Odontología 2007; 6: 131 - 134]
\end{abstract}

Palabras clave: Hipodoncia, Ausencia dental, Oligodoncia.

\section{HYPODONTIA: A FAMILIAL CASE REPORT}

\begin{abstract}
Hypodontia is defined as the ausence of one to six teeth, without considering third molares; it is an event that happens, in the permanent teething, between the $1.6 \%$ to $9.6 \%$ as much in men as in women. A case of family hypodontia is reported with a pattern of inheritance autosomal recessive, with absence of lateral incisive 22, and second premolar in the four quadrants besides the third molars and microdontia of lateral incisive 12 .
\end{abstract}

Key words: Hypodontia, Dental abcense, Oligodontia.

Recibido para publicación: 3 de septiembre de 2007. Aceptado para publicación: 26 de noviembre de 2007.

\section{INTRODUCCIÓN}

La agenesia de uno o más dientes es la anomalía más común en el desarrollo dental del hombre. La definición de hipodoncia corresponde a la falta de uno a seis dientes, sin tener en cuenta a los terceros molares. En contraste, la oligodoncia se da cuando faltan más de 6 dientes. ${ }^{1}$

La agenesia de dientes permanentes tiene una incidencia del $10 \%$ al $15 \%$ en Norteamérica, y puede ocurrir de forma aislada o como parte de un síndrome (más de 49 síndromes asociados). Los casos aislados pueden ser familiares o esporádicos. ${ }^{1}$

La agenesia de dientes familiar es trasmitida como una condición autosómica dominante, recesiva o ligada a X. Los gemelos homocigotos han mostrado una alta concordancia en hipodoncia comparados con los gemelos dicigoticos. ${ }^{1}$

Adicionalmente, varias anomalías dentales, especialmente la hipodoncia tienen alta prevalencia en ciertos grupos, como en niños con labio y/o paladar hendido, en personas con displasia ectodérmica y con síndrome de Down, Rieger y Book, entre otros. ${ }^{1}$

La anodoncia es un caso extremo que denota la ausencia completa de dientes. La anodoncia y la oligodoncia se asocian usualmente con anomalías sistémicas como las ya mencionadas y son raras, pero la hipodoncia es relativamente común.

La hipodoncia en la dentición primaria es más común en el maxilar superior y frecuentemente está asociada con el incisivo lateral. Algunos estudios siguieren que ocurre en $0.1 \%$ al $0.9 \%$ de la población, con igual frecuencia en hombres que en mujeres. Como regla, cuando falta el diente temporal, su contraparte permanente también estará ausente. ${ }^{1}$

La hipodoncia de dientes permanentes ocurre con igual frecuencia en arco superior e inferior y afecta usualmente, a los terceros molares. El tipo de diente y la prevalencia varía con cada grupo racial, aunque es más frecuente en mujeres. ${ }^{2}$ 
En muchas poblaciones se ha reportado que, con excepción del tercer molar, el diente faltante más común es el incisivo lateral superior y segundo premolar inferior. En Europa, el segundo premolar inferior es el más frecuentemente ausente después del tercer molar, seguido del incisivo lateral superior y segundo premolar superior ${ }^{2,3}$ Sin tener en cuenta el tercer molar, la prevalencia mundial varía entre 1,6 y $9,6 \%{ }^{4}$

Otros estudios muestran una mayor frecuencia de hipodoncia bilateral que unilateral y varían los hallazgos de mayor frecuencia de arco superior o inferior. Se reporta también una ausencia muy rara de incisivos centrales superiores, y primeros molares y caninos..$^{5-7}$

Algunos autores como Mckeown (2002) y Bazan (1983) han mostrado asociación en los estudios entre microdoncia e hipodoncia, mientras otros no muestran diferencias estadísticamente significativas. $^{5,8}$

La mutación heredada como patrón dominante en humanos de genes PAX9 y MSX1 ha sido identificada como causa de ausencia dental especialmente de dientes posteriores. La mutación PAX9 causa agenesia de molares permanentes y la delección completa del PAX9 se ha involucrado en la ausencia de molares temporales y permanentes. ${ }^{9}$ También se ha asociado la mutación MSX1 con agenesia de segundos premolares y terceros molares. ${ }^{9}$

Adicionalmente, la mutación de P63 humano se ha asociado a síndromes y displasia de esmalte hasta ausencia de dientes tanto temporales como permanentes. La mutación de DLX1 y DLX2 actúa con un fenotipo recíproco a la mutación de activinBA donde todos los molares están ausentes. Así mismo, la mutación de EDA1, EDAR y EDARADD se asocia con pérdida dental y malformación (displasia ectodérmica hipohidrótica). ${ }^{9}$

En los individuos con agenesia parcial asociada con mutación de MSX1, cerca de 1 de cada 5 incisivos centrales mandibulares faltan y faltan en promedio $75 \%$ de los primeros premolares superiores y solo $15 \%$ de los primeros premolares inferiores. ${ }^{10}$

En las personas con mutación en PAX9 falta su incisivo central, segundo premolar superior, primer molar superior, segundo molar superior e inferior y dientes temporales ${ }^{10}$
En estudios realizados en Colombia se determinó el patrón de herencia, la prevalencia y penetrancia y los genes involucrados en siete grupos colombianos con hipodoncia, con resultados que indican que la prevalencia de hipodoncia en el grupo de estudio fue del $13.6 \%$ con un patrón de expresión clínica variable: $46.1 \%$ para los incisivos laterales superiores, $38.4 \%$ para los terceros molares y un 9.2\% para primeros premolares. Se transmite de manera autosómica dominante con penetrancia incompleta. El análisis de restricción para MSX1 y el análisis de ligamiento en PAX9 indicaron que esta forma de hipodoncia tiene una etiología diferente $^{11-13}$

El propósito de este artículo es reportar un caso de hipodoncia familiar con patrón de herencia autosómica recesiva.

\section{REPORTE DE CASO}

Paciente de género femenino, de 11 años que consultó por la ausencia de un incisivo lateral y microdoncia de lateral presente. Se realiza un control de su proceso eruptivo hasta los 16 años. En el proceso se nota ausencia de erupción de premolares como se evidencia en la radiografía panorámica, erupción de canino en el lugar del incisivo lateral izquierdo y permanencia de $55,63,65,74$ y 84 (Figura 1). Además, se observan ausencia de los cuatro terceros molares permanentes (Figura 2, 3 y 4).

Figura 1. Radiografía panorámica donde se observa ausencia de lateral derecho superior, segundos premolares superiores e inferiores y terceros molares.

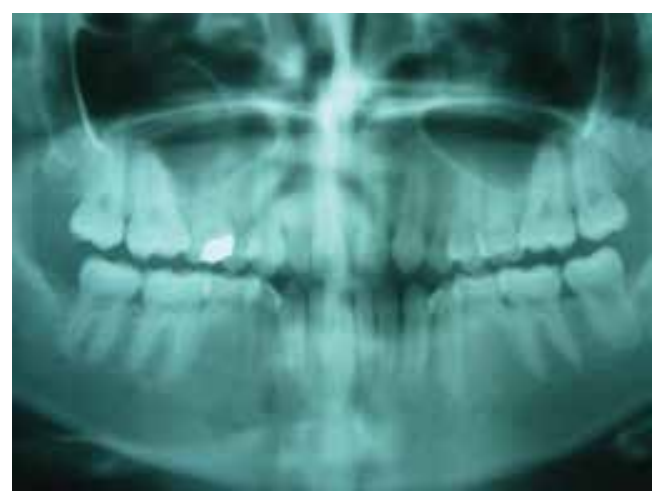

Figura 2, 3, 4. Fotografias del paciente que evidencian la ausencia de permanentes y presencia de $55,63,65,75,85$. 

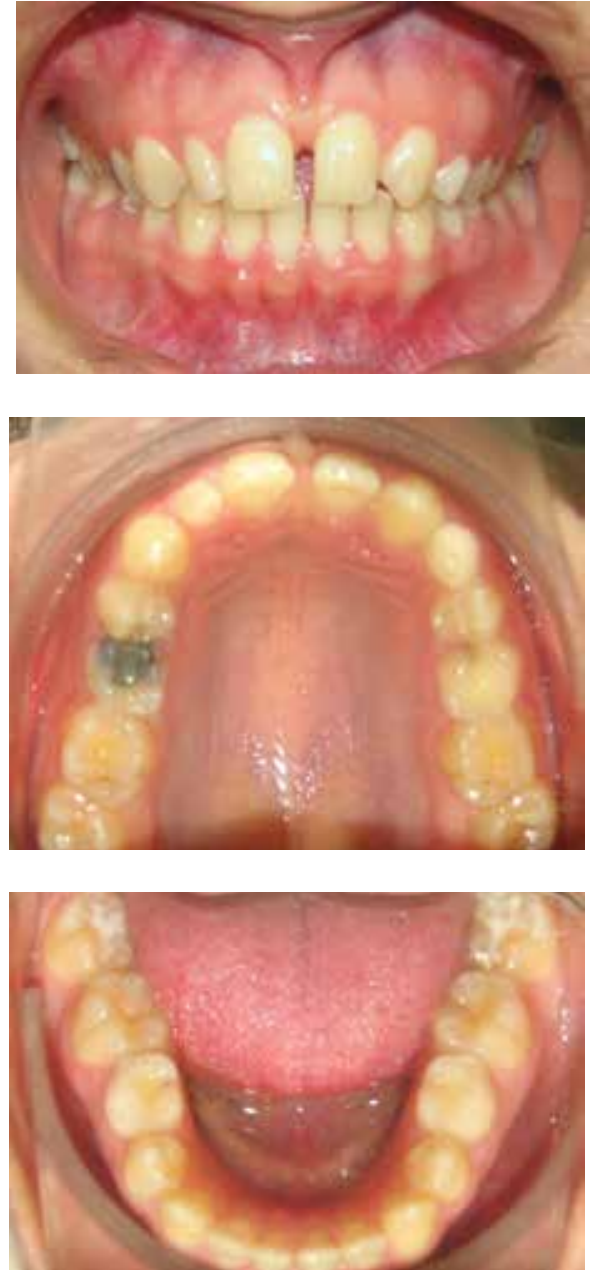

\section{Estudio familiar}

El gráfico muestra cómo la afección se ha presentado en la familia materna con cuatro individuos afectados, una madre y padre no afectados y un patrón de herencia autosómico recesivo, el individuo II-4, II-8, II-9, II-10, todos ellos con ausencia de incisivo lateral únicamente (Figura 5, 6 y 7).

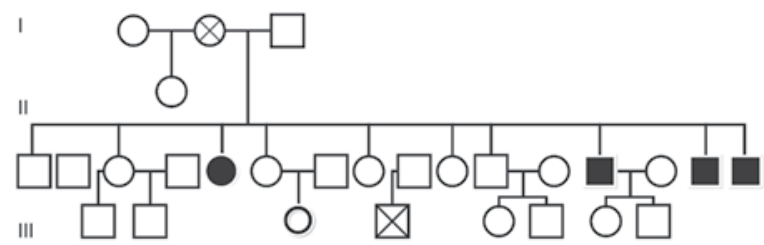

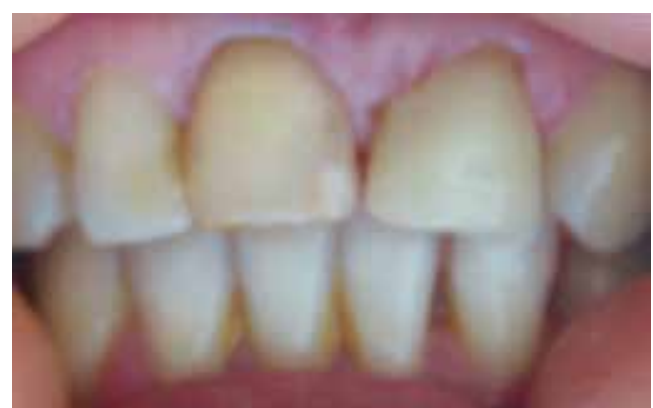

Figura 6. Individuo II-8

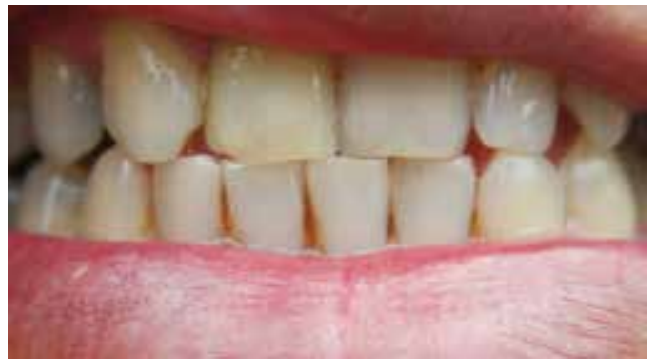

Figura 5. Individuo II-9.

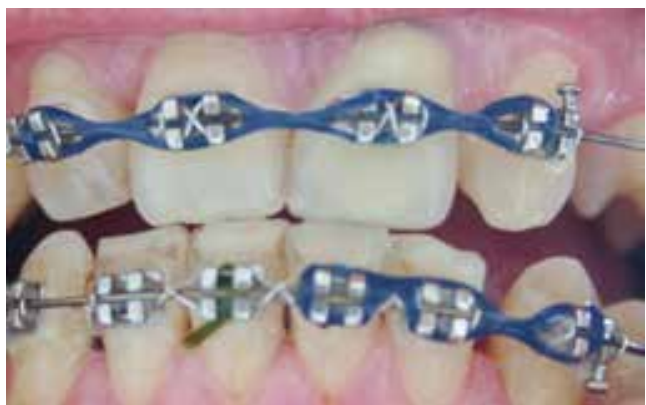

DISCUSIÓN

El carácter ausencia de dientes puede ser considerado como una variante normal en la población, pues en el proceso evolutivo se ha podido constatar que hay una tendencia a la desaparición del último diente de cada serie como adaptación a los cambios en la forma en la que se alimenta el ser humano. ${ }^{14}$ Sin embargo, este carácter puede ser aislado o familiar.

Cuando se habla de carácter familiar, se debe considerar las llamadas herencias mendelianas o monogénicas, causadas por la presencia de un solo gen mutado que determina la aparición del carácter y su transmisión de una generación a otra a través de los gametos (óvulos y espermatozoides); estos 
tipos de herencia pueden ser dominantes recesivas y autosómicas o ligadas al sexo. ${ }^{15}$

Serán autosómicas si el gen afectado se encuentra en un cromosoma no sexual, y dominantes o recesivas si el gen tiene mayor o menor capacidad de expresión. Ligadas al sexo o al cromosoma X si el gen afectado está en el cromosoma $\mathrm{X}$ que también puede ser dominante o recesivo. ${ }^{15}$

De acuerdo con los reportes en la bibliografía clásica genética y de características hereditarias, sería posible afirmar que puede aparecer en cualquiera de los cuatro patrones mendelianos, pero de acuerdo con lo revisado, es más frecuente como un rasgo autosómico dominante o recesivo, pero con bastante más predisposición a hacerse dominante con expresividad variable. ${ }^{15}$

En este estudio familiar, la oligodoncia o agenesia dentaria aparece como un carácter autosómico recesivo, pues se observa en dos generaciones consecutivas sin una relación de consanguinidad directa entre los afectados y sin predisposición con el sexo de los afectados que pueden ser hembras o varones.

El riesgo de la aparición del carácter y su expresión es variable, pues aparece en diferentes grados: leve, moderado y severo. En los casos presentados, cuatro individuos de la segunda generación presentan ausencia unilateral de un incisivo lateral superior y es considerada la expresividad de carácter leve; el caso reportado, con ausencia de nueve dientes, que incluyen la ausencia de los terceros molares.

En relación con la frecuencia de aparición en los casos estudiados, se comprobó que los laterales superiores estuvieron ausentes en cinco pacientes de manera unilateral, los segundos premolares inferiores y superiores en un caso y los cuatro terceros molares en el mismo caso.

\section{CONCLUSIONES}

1. La agenesia dentaria es una entidad patológica vista con relativa frecuencia en nuestro medio.

2. En la familia objeto de estudio se pone de manifiesto el modo de transmisión del carácter.
3. Se comprobó en esta familia que la agenesia dentaria aparece como autosómica recesivo.

4. En los cinco casos presentados se puso de manifiesto la variabilidad del carácter; en uno de los hermanos se presentó leve (un sólo diente ausente), severo en caso III-3, con nueve dientes ausentes que incluían a los terceros molares.

\section{BIBLIOGRAFÍA}

1. Hall RK. Congenitally missing teeth - A diagnostic feature in many syndromes of the head and neck. J Int Assoc Dent Child 1983; 14: 69-75

2. Jorgenson RJ. Clinician's view of hypodontia. JADA 1980: 101: $283-286$.

3. Pirinen S, Kentala A, Nieminen P, Varilo T, Thesleff I, Arte $\mathrm{S}$. Recessively inherited lower incisor hypodontia J Med Genet 2001; 38: 551 - 556

4. Fekonja A. Hypodontia in orthodontically treated children. Eur J Orthod 2005; 27: 457 - 460.

5. Kirzioglu Z, Köseler Sentut T, Ozay Ertük MS, Karayilmaz $\mathrm{H}$. Clinical features of hypodontia and associated dental anomalies: a retrospective study. Oral Diseases 2005; 11: $399-404$

6. Silva Meza R Radiographic assessment of congenitally missing teeth in orthodontic patients. Int J Paediatr Dent 2003; 13: 112 - 116 .

7. Rasmussen P. Severe hypodontia: diversities in manifestations. J Clin Pediatr Dent 1999; 23: 179 - 188.

8. McKeown HF, Robinson DL, Elcock C, Al-Sharood M,Brook $\mathrm{AH}$. Tooth dimensions in hypodontia patients, their unaffected relatives and a control group measured by a new image analysis system. Eur J Orthod 2002; 24: 131 - 141.

9. Miletich I, Sharpe PT. Normal and abnormal dental development. Hum Mol Genet 2003; 12: R69 - R73.

10. Kim JW, Simmer JP, Lin BPJ, Chu JC. Novel MSX2 frameshift causes autosomal dominant olygodontia. J Dent Res 2006; 85: 267 - 271.

11. Briceño I, Berrocal MC, González OA, Gutiérrez SJ, Ordoñez A, Torres D. Análisis clínico y molecular de la hipodoncia. Memorias IV - V Encuentro de Investigación en Ciencias Básicas en Odontología. URL disponible en: http://encolombia.com/odontologia/investigaciones/memorias-seminario.htm

12. Arboleda LA, Echeverri J, Restrepo LA, Marín ML, Vásquez G, Gómez JC, Manco HA, Pérez CM, Taborda E. Agenesia dental. Revisión bibliográfica y reporte de dos casos clínicos. Revista Facultad de Odontología Universidad de Antioquia 2006; 18: 47 - 54.

13. Bazán M. A congenitally missing canine in association with other dental disturbance: report of two cases. J Dent Child 1983; 50: 382 - 384.

14. Corrucini RS. An epidemiologic transition in dental occlusion in world populations. Am J Orthod 1984; 86: 419.

15. Correa MB, García-Robes M. Agenesia dentaria: Estudio familiar. Rev Cubana Ortod 1996;11: 27 - 34. 